\title{
The Law of Diminishing Returns in Clinical Medicine: How Much Risk Reduction is Enough?
}

\author{
James W. Mold, MD, MPH, Robert M. Hamm, PhD, and Laine H. McCarthy, MLIS
}

The law of diminishing returns, first described by economists to explain why, beyond a certain point, additional inputs produce smaller and smaller outputs, offers insight into many situations encountered in clinical medicine. For example, when the risk of an adverse event can be reduced in several different ways, the impact of each intervention can generally be shown mathematically to be reduced by the previous ones. The diminishing value of successive interventions is further reduced by adverse consequences (eg, drug-drug, drug-disease, and drug-nutrient interactions), as well as by the total expenditures of time, energy, and resources, which increase with each additional intervention. It is therefore important to try to prioritize interventions based on patient-centered goals and the relative impact and acceptability of the interventions. We believe that this has implications for clinical practice, research, and policy. (J Am Board Fam Med 2010;23: 371-375.)

Keywords: Risk Reduction, Clinical Decision-Making, Evidence-Based Medicine

In economics, the law of diminishing returns states that, as "quantities of one variable factor are increased, while other factor inputs remain constant, all things being equal, a point is reached beyond which the addition of one more unit of the variable factor will result in a diminishing rate of return and the marginal physical product will fall." ${ }^{1}$ The same phenomenon applies in a variety of clinical situations, particularly in the diagnostic process and in situations where multiple tests or treatments could be recommended for the same patient. More than 15 years ago, Johnson ${ }^{2}$ discussed the law of diminishing returns as it relates to the diagnostic process.

This article was externally peer reviewed.

Submitted 15 July 2009; revised 1 September 2009; accepted 18 September 2009.

From the Department of Family and Preventive Medicine, University of Oklahoma Health Sciences Center, Oklahoma City.

Funding: none.

Conflict of interest: none declared.

Corresponding author: James W. Mold, MD, MPH, Department of Family and Preventive Medicine, University of Oklahoma Health Sciences Center, 900 NE 10th Street, Oklahoma City, OK 73104 (E-mail: james-mold@ouhsc.edu).

\footnotetext{
See Related Commentary on Page 283.
}

The abstract of his paper begins with the following statement: "In the quest for diagnostic certainty, one can be led into a false sense of accomplishment by the results of sensitive, specific, and well-executed diagnostic tests that provide little or no diagnostic information. This is a consequence of the fact that as one approaches diagnostic certainty the useful information returned by diagnostic tests and observations approaches zero." 2 The purpose of this article is to explore the clinical implications of the law of diminishing returns for risk-reduction strategies (prevention of future adverse events).

Clinical applications of the law of diminishing returns have been mentioned by several authors. Sonnenberg, ${ }^{3}$ writing about gastrointestinal interventions, wrote that the physician "has to weigh the benefit and harm of each sequential medical intervention and decide how far to extend the therapeutic chain and how much longer to proceed in finetuning the patient's health." Luke and colleagues, ${ }^{4}$ discussing the benefits and harms associated with maternal weight gain, wrote, "These findings suggest that, beyond a certain level of weight gain, there is a point of diminishing returns (increase in birth weight) at the expense of increasing maternal postpartum obesity..." Probably the best way to illustrate the principle of diminishing returns as it applies to risk reduction is with a case. 


\section{Case}

Mr. Martin is a fictional, 65-year-old, AfricanAmerican man who was diagnosed a year ago with type 2 diabetes mellitus. He leads a sedentary life but does not smoke or drink alcohol to excess. So far, he has had no clinically apparent end organ complications from his diabetes. However, he has recently noticed fatigue, polyuria, and polydipsia. He currently takes no medications. His past and family history are otherwise unremarkable. At the insistence of his wife he agreed to see his primary care physician, who finds that his body mass index is 30.5 , his blood pressure is $200 / 100 \mathrm{~mm} \mathrm{Hg}$, his hemoglobin A1c (A1c) is 10\%, his low-density lipoprotein (LDL) cholesterol is $140 \mathrm{mg} / \mathrm{dL}$, his high-density lipoprotein cholesterol is $40 \mathrm{mg} / \mathrm{dL}$, and his total cholesterol is $260 \mathrm{mg} / \mathrm{dL}$.

\section{Risk Reduction}

Mr. Martin should, according to American Diabetes Association guidelines, be advised to lose weight, exercise moderately, take low-dose aspirin, lower his blood pressure to below 130/80, lower his LDL level to below $100 \mathrm{mg} / \mathrm{dL}$, reduce his A1c to below $7 \%$, take an angiotensin-converting enzyme inhibitor, and possibly take a $\beta$-blocker to reduce his risk of a myocardial infarction (MI). ${ }^{5}$ Each of these interventions-other than weight loss and A1c reduction-has been shown, in separate clinical trials, to lower the risk of MI by between 5\% and $35 \%$.

For simplicity, let us assume that each intervention independently lowers the risk of MI by $20 \%$, and that our patient's baseline absolute risk (AR) of $\mathrm{MI}$ is $36 \%$ during the next 10 years. Mathematically, the first intervention would reduce his risk of MI by $20 \%$ and his AR of an MI by $7.2 \%$ (20\% of $36 \%=7.2 \%)$. Because his MI risk has now been reduced to $28.8 \%(36 \%-7.2 \%)$, the second intervention would lower his AR by only $5.8 \%$ (20\% of $28.8 \%$ ). The third, fourth, and fifth interventions would lower his AR by $4.6 \%, 3.7 \%$, and $2.9 \%$, respectively. The combined benefit of all 6 interventions would result in an absolute risk reduction (ARR) of $26.6 \%$, or a new AR of MI of $9.4 \%$. The first 3 interventions were responsible for two thirds of this reduction $(7.2 \%+5.8 \%+$ $4.6 \%=17.6 \% ; 17.6 \div 26.6=66.1 \%)$. The mathematical equation for this is:
Table 1. Individual and Cumulative Absolute Risk Reductions of Interventions on 10-Year Risk for Myocardial Infarction for Mr. Martin from the Archimedes Risk Calculator*

\begin{tabular}{lccc}
\hline Interventions & $\begin{array}{c}\text { Individual } \\
\text { ARRs (\%) }\end{array}$ & $\begin{array}{c}\text { Sequential } \\
\text { ARRs (\%) }\end{array}$ & $\begin{array}{c}\text { Risk } \\
(\%)\end{array}$ \\
\hline Aspirin & 13.5 & 13.5 & 22.9 \\
Lower SBP to 130 & 7 & 4.1 & 18.8 \\
Moderate exercise & 6.8 & 5.4 & 13.4 \\
B-blocker & 4.5 & 0.4 & 13.0 \\
ACE inhibitor & 2.9 & 1.1 & 11.9 \\
Lower LDL to 100 & 2.6 & 0.2 & 11.7 \\
\hline
\end{tabular}

*Base risk, 36.4\%.

ARR, absolute risk reductions; SBP, systolic blood pressure; ACE, angiotensin-converting enzyme; LDL, low-density lipoprotein.

$$
A R R=\sum_{i=1}^{n}\left(R_{i-1} \times R R R_{i}\right)
$$

where $\mathrm{R}_{i-1}$ is the risk before intervention $i$, and $\mathrm{RRR}_{i}$ is the relative risk reduction expected from intervention $i$. As $\mathrm{R}_{i-1}$ grows progressively smaller, so does the impact of each intervention on ARR. Another way to think about this is that the number needed to treat equals $100 \% / A R R$. As the ARR decreases with successive interventions, the number needed to treat to prevent $1 \mathrm{MI}$ increases.

Because some interventions are more effective than others, application of the 2 most effective interventions often achieves most of the benefit that could be achieved with all interventions combined. Our assumption that the interventions act independently is also generally not correct. The mechanisms by which interventions impact risk may be similar and therefore duplicative. For example, the effects of 2 different inhibitors of platelet aggregation, such as aspirin and clopidogril, though acting at different receptor sites, are largely, but not entirely, duplicative because both reduce the risk of clotting. The effect of adding clopidogril to aspirin is therefore less than additive.

Based upon projections obtained from Diabetes Personal Health Decisions (Archimedes) ${ }^{6}$ a sophisticated and surprisingly accurate risk engine, ${ }^{7,8}$ the predicted benefits of interventions for reducing heart attack risk for Mr. Martin are shown in Table 1. In this simulation, the 3 most effective interventions-aspirin, blood pressure reduction, and aerobic exercise-reduced MI risk from 36.4\% to 
Figure 1. Projected 20-year risks of stroke for Mr. Martin as systolic blood pressure is lowered by $20 \mathrm{~mm} \mathrm{Hg}$ at a time. From the Archimedes Risk Calculator. Base risk, 62.4\%.

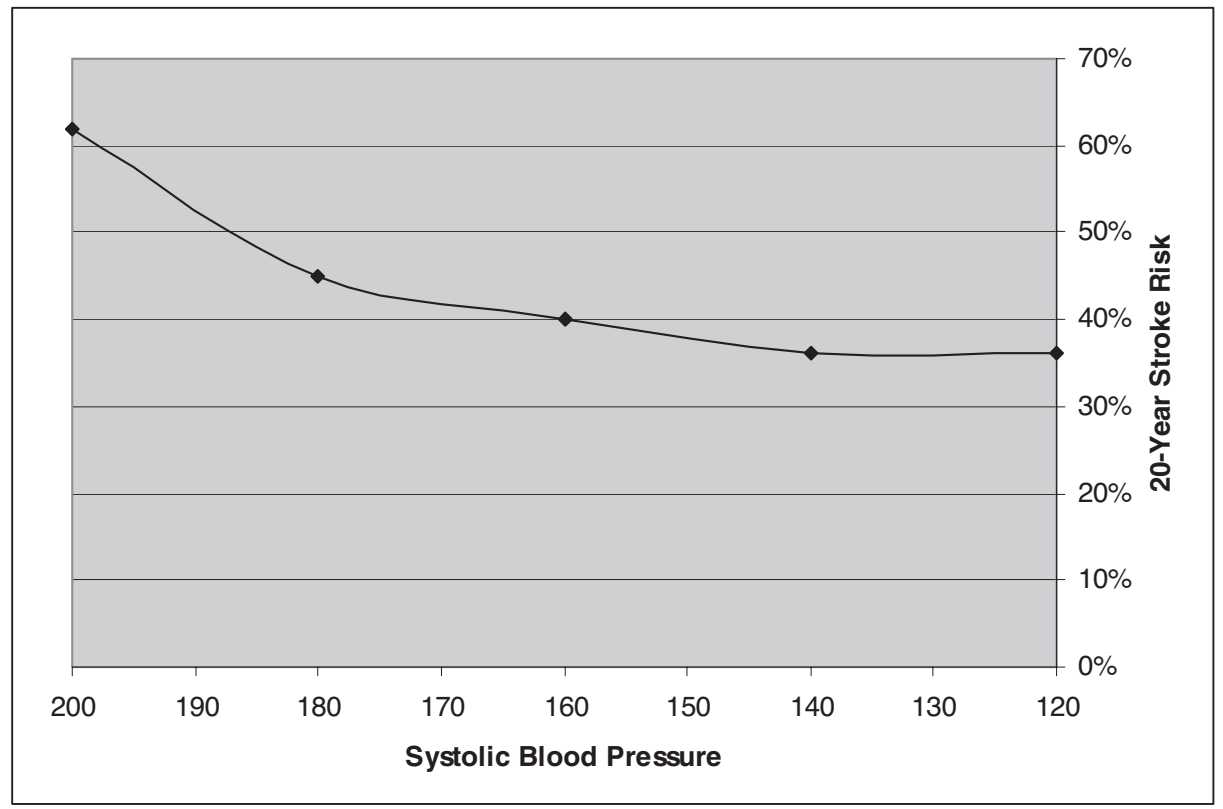

$13.4 \%, 93 \%$ of the reduction in risk that was achieved by all 6 interventions combined. Although harder to quantify because of a lack of risk prediction tools, the same effect is likely to apply to any adverse event for which multiple nonindependent risk reduction strategies are available. Examples include hospitalization for systolic heart failure (angiotensin-converting enzyme inhibitor, $\beta$-blocker, digoxin, loop diuretics, spironolactone, cardiac rehabilitation, patient education, monthly nurse phone calls); hip fracture (balance exercises, lower extremity strengthening exercises, avoidance of psychoactive medications, vitamin $\mathrm{D}$, calcium, bisphosphonates); migraine (avoidance of dietary triggers, stress reduction, high-dose riboflavin, feverfew, $\beta$-blocker, amitriptyline, topiramate, etc); and pneumonia (good oral hygiene, hand washing, pneumococcal vaccination, influenza vaccination, smoking cessation, adequate sleep, adequate hydration, avoidance of proton pump inhibitors and histamine 2 blockers, etc).

Some potentially effective risk reduction strategies may not be identified or may be discredited because of widespread use of other, possibly more expensive and hazardous ones. Recently, 2 randomized, controlled trials have suggested that low-dose aspirin may not reduce the risk of cardiovascular events very much in patients with type 2 diabetes mellitus. ${ }^{9,10}$ These results seem to be in conflict with prior primary and secondary prevention trials in which aspirin was efficacious for high-risk patients. ${ }^{11}$ At least some of the apparent loss of effectiveness, as Belch et $\mathrm{al}^{9}$ point out, may be because other cardiac risk factors had already been controlled by participants in these trials. In fact, in their trial, the average total cholesterol levels of participants was almost 30\% lower than in studies of a similar population 10 years earlier, presumably because of increased use of statins. Average systolic blood pressures were relatively low (143) because a high proportion of participants were probably being treated with angiotensin-converting enzyme inhibitors. Thus, the ARRs remaining to be reduced by aspirin may have been too small to detect.

The law of diminishing returns may also apply within risk factors. For example, lowering systolic blood pressure (SBP) from 200 to 180 may reduce stroke risk more than lowering it from 180 to 160. ${ }^{12}$ Figure 1 shows the Archimedes projections for the impacts of incremental reductions in SBP on 20-year risk of stroke for Mr. Martin. Similarly, lowering A1c from 10 to 9 may reduce the risk of microvascular complications more than lowering it from 8 to 7 , even though reductions in the lower ranges are proportionately larger. ${ }^{13-18}$ The Action to Control Cardiovascular Risk in Diabetes trial showed that bringing average blood sugars too 
close to normal in older patients may actually result in worse outcomes. ${ }^{19}$

Still, it could be argued that we should do everything humanly possible to reduce the risk of future adverse events. There are several other good reasons not to do so. First, the larger the number of medications prescribed, the less likely they will be taken consistently and according to directions. ${ }^{20-22}$ In addition, the costs and the number of side effects increase linearly with each additional medication, and the number of potential drug-drug, drug-food, and drug-disease interactions increases exponentially. As a rule of thumb, once the number of medications reaches approximately 5 , the probability of adverse events becomes high enough to counterbalance any positive effects of additional medications. $^{22}$

Unfortunately, the list of recommended interventions grows as a multiple of the number of guidelines because each guideline includes multiple recommendations. Boyd and colleagues ${ }^{23}$ applied disease-oriented clinical practice guideline recommendations to a simulated 79-year-old woman with 5 common chronic diseases: chronic obstructive pulmonary disease, type 2 diabetes mellitus, osteoporosis, hypertension, and osteoarthritis. Recommended interventions included, conservatively, 12 different medications taken at 5 different times during the day at a cost (for generic brands) of $\$ 406$ per month. Multiple potential drug-drug interactions were identified. ${ }^{23}$

\section{Prioritization}

Clinicians and patients must recognize that some risk reduction strategies are more effective than others and that it is often not possible to expect patients to embrace and adhere to all of them. The law of diminishing returns provides reassurance that, once the most effective interventions have been instituted, the rest will probably be of little benefit and are likely to increase cost and cause harm. The most reasonable way, then, to apply the law in clinical medicine is to help patients choose from among the available interventions the 2 or 3 most likely to achieve the desired outcomes (eg, life prolongation, ability to drive, etc). ${ }^{24,25}$

\section{Research Implications}

At present, rational prioritization is difficult because of the lack of pertinent information. ${ }^{26}$ Clin- ical studies most often examine the efficacy of single tests or treatments, not sequential ones. Effect sizes across the range of single risk factors are rarely calculated or reported. The law of diminishing returns therefore provides additional justification for comparative effectiveness research. We need more sophisticated decision support tools for patients and clinicians that collect risk factors, link them to available interventions, and stratify the interventions based on the size of their impact on outcomes. Armed with such tools, patients and their clinicians would be better able to select the interventions that would probably produce the most benefit while considering the personal costs and difficulties of each.

\section{Policy Implications}

In a time of increasing concerns about cost containment, the law of diminishing returns coupled with the concept of value could provide a rational framework within which to discuss spending restraints both in terms of guideline development and newer technologies. Value can be defined as benefit divided by cost (value $=$ benefit $\div$ cost) ${ }^{27,28}$ As discussed above, increasingly aggressive risk reduction interventions tend to be associated with both smaller and smaller benefits and exponentially increasing costs. For example, Jacobson ${ }^{29}$ observed, "In light of the current climate involving competing health care costs, the pursuit of progressively diminishing returns in terms of reductions in coronary artery disease risk through more aggressive lowering of LDL cholesterol levels appears to be unwarranted." Learmonth, ${ }^{30}$ addressing the cost of developing better and better hip prostheses, stated, "[D] evelopments in the science and surgery of total hip arthroplasty have delivered a very successful surgical procedure. However, the profession and industry will not stand still...The law of diminishing returns applies. As the product becomes more successful, an exponentially greater investment is required to achieve further improvement."

\section{Conclusions}

In the context of an enlarging armamentarium of interventions providing both benefits and harms and escalating health care costs, the law of diminishing returns could provide a framework for rational prioritization. Clinicians are urged to consider both sequencing and independence of 
effect when recommending risk reduction strategies. Policymakers may find the concept useful, within the context of value, when framing discussions about cost constraints. Researchers are encouraged to find ways to provide the kinds of information needed by clinicians and patients who are trying to make rational choices about available interventions.

\section{References}

1. Economy Professor. Law of diminishing returns. The Professor Network, 2009. Available at www. economyprofessor.com/economictheories/law-ofdiminishing-returns.php. Accessed 14 April 2009.

2. Johnson HA. Diminishing returns on the road to diagnostic certainty. JAMA 1991;265:2229-31.

3. Sonnenberg A. Diminishing returns on sequential interventions of gastroenterology. Eur J Gastroenterol Hepatol 2008;20:465-8.

4. Luke B, Hediger ML, Scholl TO. Point of diminishing returns: when does gestational weight gain cease benefiting birthweight and begin adding to maternal obesity? J Matern Fetal Med 1996;5:16673.

5. American Diabetes Association. Standards of medical care in diabetes-2008. Diabetes Care 2008; 31(Suppl 1):S12-S54.

6. American Diabetes Association. Living with diabetes. Diabetes PHD. Available at http://www.diabetes. org/living-with-diabetes/complications/diabetes-phd/. Accessed 15 March 2010.

7. Eddy DM, Schlessinger L. Validation of the Archimedes diabetes model. Diabetes Care 2003;26: 3102-10.

8. Eddy DM, Schlessinger L. Archimedes: a trial-validated model of diabetes. Diabetes Care 2003;26: 3093-101.

9. Belch J, MacCuish A, Campbell I, et al. The prevention of progression of arterial disease and diabetes (POPADAD) trial: factorial randomized placebo controlled trial of aspirin and antioxidants in patients with diabetes and asymptomatic peripheral arterial disease. BMJ 2008;337:a1840.

10. Ogawa H, Nakayama M, Morimoto T, et al. Lowdose aspirin for primary prevention of atherosclerotic events in patients with type 2 diabetes. JAMA 2008;300:2134-41.

11. US Preventive Services Task Force. Aspirin for the primary prevention of cardiovascular events. Report no. APPIP02-0004. Washington, DC: Agency for Healthcare Research and Quality.

12. Kannel WB, Schatzkin A. Risk factor analysis. Prog Cardiovasc Dis 1983;26:309-32.

13. Gerstein HC, Pogue J, Mann JFE, et al. The relationship between dysglycaemia and cardiovascular and renal risk in diabetic and non-diabetic partici- pants in the HOPE study: a prospective epidemiological analysis. Diabetologia 2005;48:1749-55.

14. Haffner SM. Is there a glycemic threshold?. Arch Intern Med 1997;157:1791.

15. Krolewski AS, Laffel LMB, Krolewski BA, Quinn M, Warram JH. Glycosylated hemoglobin and the risk of microalbuminaria in patients with insulin-dependent diabetes mellitus. N Engl J Med 1995;332:1251-5.

16. Orchard TJ, Forrest KY-Z, Ellis D, Becker D. Cumulative glycemic exposure and microvascular complications in insulin-dependent diabetes mellitus: the glycemic threshold revisited. Arch Intern Med 1997;157:1851-6.

17. Vijan S, Hofer TP, Hayward RA. Estimated benefits of glycemic control in microvascular complications in type 2 diabetes. Ann Intern Med 1997;127:788-95.

18. Warram JH, Manson JE, Krolewski AS. Glycosylated hemoglobin and the risk of retinopathy in insulin-dependent diabetes mellitus. $\mathrm{N}$ Engl J Med 1995;332:1305-6.

19. Action to Control Cardiovascular Risk in Diabetes Study Group (ACCORD), Gerstein HC, Miller ME, et al. Effects of intensive glucose lowering in type 2 diabetes. N Engl J Med 2008;358:2545-59.

20. Courtney DL. Medication reduction strategies. Compr Ther 1996;22:318-23.

21. Stoehr GR, Lu S-Y, Lavery L, Vander Bilt J, Saxton JA, Chang C-C. Factors associated with adherence to medication regimens in older primary care patients: the Steel Valley Seniors Survey. Am J Geriatr Pharmacother 2008;6:255-63.

22. Williams AF, Manias E, Walker R. Adherence to multiple, prescribed medications in diabetic kidney disease: a qualitative study of consumers' and health professionals' perspectives. Int J Nurs Stud 2008;45:1742-56.

23. Boyd CM, Darer J, Boult C, Fried LP, Wu AW. Clinical practice guidelines and quality of care for older patients with multiple-co-morbid diseases: implications for pay for performance. JAMA 2005;294:716-24.

24. Mold JW. An alternative conceptualization of health and health care: its implications for geriatrics and gerontology. Educ Gerontol 1995;21:85-101.

25. Mold JW, Blake GH, Becker LA. Goal-oriented medical care. Fam Med 1991;23:46-51.

26. Mold JW, Hamm R, Scheid D. Evidence-based medicine meets goal-directed health care. Fam Med 2003;35:360-4.

27. Lee TH. "Me-Too" products—friend or foe? N Engl J Med 2004;350:211-2.

28. Zubialde JP, Mold JW. Relational value: bridging the worldview gap between patients and health systems. Fam Med 2001;33:393-8.

29. Jacobson TA. "The lower the better" in hypercholesterolemia therapy: a reliable clinical guideline? Ann Intern Med 2000;133:549-54.

30. Learmonth ID. Total hip replacement and the law of diminishing returns. J Bone Joint Surg 2006;88-A: $1664-73$. 